\title{
Sensor fabrications using inkjet distributions and analysis systems
}

\begin{abstract}
Alternative for electrode fabrications were demonstrated. Interdigitated electrodes (IDE) on polyethylene terephthalate (PET) substrate using silver conductive ink were successfully fabricated. Nano-particle based conductive inks (Jet-600 C) which have wide range viscosity control were used in this study. Inkjet Distributions and Analysis Systems (IJDAS) is inkjet printing technology that allows for deposition of versatile thin films, the design of which can be changed from time to time. Printing method was using dropped-on-demand inkjet depends on the nozzles arranged in a single line. It has been shown that the silver ink with viscosity between 7 to $10 \mathrm{cps}$ is suitable for IDE fabrications on PET.
\end{abstract}

Keyword: Silver ink; Interdigitated electrode; PET substrate; Inkjet printing 